\title{
Avaliação da Reprodutibilidade e Validade de Questionário de Atividade Física para Gestantes
}

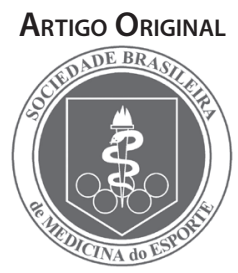

\section{Evaluation of the Reproducibility and Validity of a Physical Activity Questionnaire for Pregnant Women}

\author{
Monica Yuri Takito ${ }^{1}$ \\ Lenycia de Cassya Lopes Neri ${ }^{2}$ \\ Maria Helena, D’Aquino Benício² \\ 1. Escola de Artes, Ciências e \\ Humanidades da Universidade de \\ São Paulo \\ 2. Departamento de Nutrição da \\ Faculdade de Saúde Pública da \\ Universidade de São Paulo
}

Endereço para correspondência: Av. Prof. Mello Moraes, 65 CEP 05508-900 - São Paulo -SP Email: mytakito@gmail.com

Submetido em 28/09/2006 Versão final recebida em 05/02/2007 Aceito em 23/12/2007

\begin{abstract}
RESUMO
Introdução: A prática de atividade física tem sido incentivada por vários organismos nacionais e internacionais visando a promoção de saúde. Entretanto não existe consenso acerca da influência e da intensidade ótima de atividade física durante a gestação, possivelmente devido a dificuldades de mensuração e classificação do nível de atividade física através de questionários. Não se identificou qualquer estudo de validação de questionário de atividade física em gestantes no Brasil. O objetivo do presente estudo é analisar a reprodutibilidade e validade concorrente de um questionário de atividades físicas para gestantes. Métodos: Em amostra de 68 gestantes, no segundo trimestre de gestação foi realizada entrevista para aplicação do questionário, que foi repetida com aproximadamente uma semana de intervalo e no puerpério. No período entre as duas primeiras entrevistas, as mulheres utilizaram monitor de freqüência cardíaca ( $n=38$ ). Resultados: As análises de reprodutibilidade foram adequadas com coeficientes de correlação intraclasse variando de 0,54 a 0,85. A análise das variáveis categóricas, com questões subjetivas e de autopercepção da atividade física, resultou em maior intervalo de coeficiente kappa com valores entre 0,29 e 0,76 entre a aplicação do questionário com uma semana de intervalo, e 0,08 a 0,70 comparado ao puerpério. As análises de validade conduziram a resultado satisfatório quanto à média das diferenças encontrada por intermédio do gráfico de Bland e Altman (1986) entre o questionário e o freqüencímetro. Entretanto, o intervalo da dispersão não se apresentou adequado, visto que variou em mais de sete horas para atividades leves e em quase onze horas por dia para atividades moderadas. As análises do presente estudo parecem assegurar a reprodutibilidade do questionário de atividade física para gestantes. No entanto, com relação à validação, a comparação com os resultados obtidos pelo freqüêncímetro não mostrou graus de concordância adequados.
\end{abstract}

Palavras-chave: atividade cotidiana, confiabilidade, questionário, gravidez, cuidado pré-natal.

\section{ABSTRACT}

Introduction: Physical activity practice has been encouraged by several national and international entities aiming health promotion. However, there is no consensus concerning the influence and optimal intensity of physical activity during pregnancy, probably due to difficulties to measure and classify the level of physical activity by questionnaires. It has not been identified any validation study of physical activity questionnaire on Brazilian pregnant women. The purpose of the present study is to analyze the reliability and concurrent validity of a physical activity questionnaire for pregnant women. Methods: A sample of 68 women in the second trimester of pregnancy has been interviewed by the elaborated questionnaire. Reliability was assessed after approximately a week and in the postpartum. The subjects used a heart rate monitor between the two first interviews ( $n=38$ ). Results: Reproducibility analyses were adapted with interclass correlation coefficients varying from 0.54 to 0.85 . The analysis of categorical variables, with subjective questions of self-perception of physical activity, resulted in wider range of kappa coefficients, with values between 0.29 and 0.76 for application of the questionnaire within one week of interval, and between 0.08 and 0.70 when compared with the postpartum. The validity analyses had a satisfactory result considering the average of differences seen through Bland-Altman graphics comparing questionnaire and the heart rate monitors. However, the dispersion interval was not adequate, since it varied in more than seven hours for light activities and almost eleven hours per day for moderate activities. Analyses of current study seem to assure the reliability of the physical activity questionnaire for pregnant women. However, concerning validation, the comparison with results obtained by heart rate monitors did not show adequate degrees of agreement.

Keywords: daily activities, reliability, questionnaire, pregnancy, prenatal care. 


\section{INTRODUÇÃO}

A prática de atividade física vem sendo incentivada por vários organismos internacionais e pelo Ministério da Saúde para promoção da saúde e melhoria da qualidade de vida da população ${ }^{(1,2)}$. Dentre as iniciativas nacionais, destaca-se o Programa Agita São Paulo considerado pela OMS um modelo para os demais países ${ }^{(3)}$. A Organização Mundial da Saúde(1) define como inatividade física ter pouca ou nenhuma atividade física (não atingir 150 minutos semanais) nas situações de trabalho, transporte, tarefas domésticas ou horas de lazer. A prevalência de inatividade física entre mulheres do Município de São Paulo é igual a 40,6\%, tendo como ponto de corte menos de 150 minutos semanais de atividade moderada ou vigorosa ${ }^{(4)}$.

Artal e O'Toole ${ }^{(5)}$ enfatizam a importância da promoção da atividade física no período gestacional dada a maior possibilidade de modificação para um estilo de vida mais ativo que implicaria melhores condições de saúde da população feminina. No entanto, não existe consenso acerca da intensidade ótima da atividade física durante a gestação. Questionamentos feitos às recomendações de 1984 do Colégio Americano de Obstetras e Ginecologistas ${ }^{(6,7)}$ ocasionaram modificações que resultaram em recomendação diferenciada, segundo o condicionamento físico anterior à gestação. Assim, as mulheres menos condicionadas devem realizar atividades de menor intensidade e as mulheres fisicamente mais ativas podem manter o exercício físico, com segurança, numa intensidade superior às demais. A maior parte dos estudos foi conduzida em países desenvolvidos, em que a mulher mantém a prática de exercícios físicos e atividades esportivas durante a gestação. São poucos os estudos que enfocam a atividade física praticada por gestantes de baixo nível socioeconômico ${ }^{(8,9)}$.

Em virtude disso, torna-se importante a adequada identificação dos níveis da prática habitual de atividade física nesse segmento da população, e posterior associação de seu efeito sobre a saúde materno-fetal.

Vários métodos visando obter indicadores associados à prática de atividade física adequados à estimativa do gasto energético individual, tais como, calorimetria direta ou indireta, água duplamente marcada, freqüência cardíaca, sensores de movimento e observação direta, podem ser inviabilizados quando a análise envolve grande número de sujeitos. Quando a preocupação é avaliar grandes grupos populacionais, são fundamentais instrumentos com boa precisão, fácil aplicação e baixo custo. Dentre os instrumentos de avaliação da atividade física que utilizam informações fornecidas pelas pessoas (questionários, entrevistas e diários), destaca-se o questionário como instrumento que oferece dados de duração, freqüência, intensidade e tipo da atividade. Nesse sentido, vários estudos têm sido desenvolvidos procurando validar questionários de atividade física em diferentes grupos populacionais (crianças, adultos e idosos) $^{(10-14)}$.

Identificou-se na literatura, três estudos que propõem questionários de atividade física em gestantes. Wildshut et al.(15), em estudo exploratório com 100 gestantes inglesas, avaliaram a atividade física, incluindo atividade doméstica, durante o lazer e no trabalho, bem como a percepção do esforço. Chasan-Taber et al. ${ }^{(16)}$ validaram um questionário auto-preenchido (Pregnancy Physical Activity Questionnaire) com 54 gestantes norte-americanas. Como critério de validação concorrente utilizaram o sensor de movimento (acelerômetro uniaxial). Um terceiro instrumento, Kaiser Physical Activity Questionnaire para mulheres foi adaptado e validado com 61 gestantes, propondo classificar as gestantes segundo um escore de atividade física. A proposta de Schmidt et al. ${ }^{(17)}$ tem como intuito utilizar um mesmo instrumento para avaliar a atividade física antes e durante a gestação.

Esses questionários foram elaborados em países em desenvolvi- dos e abarcam as atividades físicas e esportivas de lazer, raramente realizadas por mulheres de baixo nível sócio-econômico, nas quais predominam atividades domésticas e ocupacionais. Também deve-se considerar as diferenças sócio-econômicas e culturais na elaboração do instrumento, indicando a necessidade de validação de um instrumento adequado as características da população estudada. Visto que no Brasil, não foram encontrados estudos de validação de questionário de atividade física para gestantes. O objetivo do presente estudo é analisar a reprodutibilidade e validade concorrente de um questionário que permita quantificar a atividade física de gestantes usuárias de serviços públicos de saúde do Município de São Paulo.

\section{METODOLOGIA}

\section{Amostra}

Foram incluídas no estudo uma amostra intencional de gestantes, contactadas em três serviços públicos de atendimento pré-natal - estando um desses inserido em uma maternidade público de grande porte e duas unidades básicas de saúde, no segundo trimestre de gestação, em decorrência do fato de que nesse período já são conhecidos os fatores de risco da mulher e ainda não houve um aumento de peso que impossibilite a realização de atividades físicas. A idade gestacional foi avaliada pela data da última menstruação, confirmada por ultrassonografia anterior a 20ª semana. Foram critérios de exclusão do estudo: gestantes com idade inferior a 18 anos, com morbidade potencialmente associada à alteração da atividade física habitual e não alfabetizadas.

O planejamento do estudo previa uma amostra de 40 mulheres no período de 2 a 3 meses, contando com 4 equipamentos e não prevendo tantas recusas e exclusões. Entretanto, foram necessários 13 meses para alcançar 34 gestantes com dados completos de questionário (teste e reteste), diário de atividades físicas e freqüência cardíaca armazenada durante o tempo solicitado (4 dias). Ainda que o tamanho da amostra tenha variado de acordo com tipo de análise, o menor valor $(n=34)$ permitiu um poder de teste de 90\% para detectar um coeficiente de correlação de 0,50, com nível de significância de 5\%(18).

\section{Desenho do estudo}

As informações foram obtidas mediante entrevistas com duração média de 31 minutos, realizadas em duas ocasiões, com intervalo de cinco dias, sendo a segunda entrevista (reteste) efetuada para verificação da reprodutibilidade do questionário.

O questionário avaliado foi elaborado com base nos questionários mencionados a seguir:

- Questionário de Wildschut et al(15), modificado segundo sugestão dos próprios autores incluindo questões relacionadas às atividades domésticas;

- Suplemento do Minnesota Leisure-Time Physical Activity Questionnaire (LPTA) / Household Activities ${ }^{(19)}$ : engloba atividades domésticas;

- International Physical Activity Questionnaire (IPAQ) versão longa(20), desenvolvido com o apoio da Organização Mundial da Saúde por representantes de 25 países, para estimar a prática habitual de atividade física de populações de diferentes países e contextos sócio-culturais. Incluiu-se no questionário duas questões visando verificar a percepção das gestantes quanto à intensidade de atividades físicas vigorosas e moderadas.

A freqüência cardíaca é considerada indicador fisiológico da atividade física, pois possui relação linear com o consumo de oxigênio para muitas atividades ${ }^{(21)}$. Embora a freqüência cardíaca não seja um 
método preciso para estimativa do gasto energético para todas as atividades diárias, em especial as atividades leves, ela pode indicar a elevação da intensidade na realização da atividade física. Com base no exposto e considerando também a disponibilidade do equipamento foi utilizado o freqüêncímetro Polar ${ }^{\circledR}$, modelo S 610i, para mensuração da freqüência cardíaca minuto a minuto. Dessa maneira, após a entrevista, as gestantes foram instruídas quanto à utilização do monitor de freqüência cardíaca (durante quatro dias) e ao preenchimento do diário de atividades físicas. Esse foi proposto para melhor compreensão da relação entre a freqüência cardíaca e as atividades realizadas. As gestantes foram orientadas a não alterar as rotinas diárias. A orientação quanto ao uso do freqüêncímetro incluiu a colocação da cinta, a ligação e verificação da efetiva gravação, bem como a retirada do equipamento para dormir e tomar banho.

O estudo foi planejado visando facilitar ao máximo a participação das gestantes. Como muitas não poderiam retornar ao serviço de saúde para devolução dos aparelhos, optou-se por visitas domiciliares para realização dos questionários e monitoração do uso do aparelho e preenchimento do diário, minimizando assim, falhas na coleta de dados. Mesmo quando detectou-se falhas no preenchimento do diário de atividades físicas ou mal uso do equipamento, o questionário foi reaplicado e as gestantes permaneceram no estudo de reprodutibilidade.

O diagrama a seguir expressa as ações realizadas para a coleta de dados deste estudo:

Informação dos procedimentos e concordância em participar (TCLE).

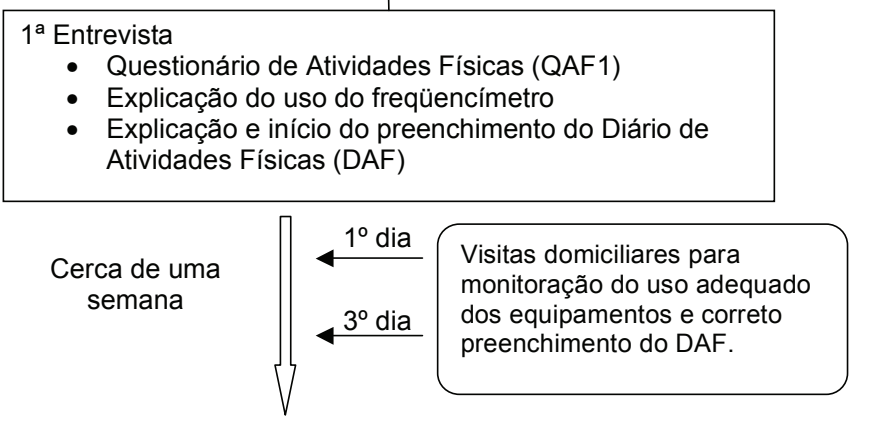

$2^{\mathrm{a}}$ Entrevista

- Reteste QAF2

- Devolução dos equipamentos e do DAF

Monitoração do nascimento através de telefonemas a residência da gestante, próximo à data estimada do parto.

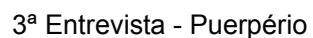 \\ - QAF3 \\ - MAQ adaptado}

No questionário foram avaliadas as atividades físicas desenvolvidas no trabalho doméstico, no trabalho fora de casa, no lazer e para a locomoção. O tempo despendido em cada atividade foi obtido através da multiplicação da freqüência e duração, expresso em minutos ou horas por dia. Foi utilizado o Compêndio de Atividades Físicas ${ }^{(22)}$ para quantificar o esforço em MET (equivalente metabólico de repouso) em cada atividade questionada. Inicialmente, as atividades foram agrupadas segundo a intensidade: sedentárias (<1,5 METs), leves (1,5-2,9 METs), moderadas (3,0-6,0 METs) e vigorosas (>6,0 METs). As atividades também foram agrupadas pelo tipo: ocupacional, domésticas, locomoção e lazer e sedentárias ${ }^{(16)}$. E ainda pelas posturas predominantes: permanência na postura ereta e agachada. O tempo e os METs despendidos diariamente foram calculados. Para os dados mensurados da freqüência cardíaca a cada minuto, a estimativa do MET foi obtida por intermédio de equação específica para gestantes, no segundo trimestre, apresentada por Pirvanik et al. ${ }^{(7)}$.

O questionário foi duplamente digitado e corrigido, utilizando o programa Epi Info versão 6.04. A transcrição dos diários de atividades físicas foi duplamente conduzida e comparada para efetiva codificação e classificação das atividades.

\section{Análise estatística}

Para as análises estatísticas utilizou-se o programa estatístico Stata versão 7.0. A análise de reprodutibilidade foi conduzida utilizandose o coeficiente de concordância Kappa para variáveis qualitativas nominais ${ }^{(23)}$ e o coeficiente de correlação intraclasse para variáveis quantitativas pela análise de variância (ANOVA) com dois fatores (aleatório: gestante e fixo: momento). A seguir foi efetuada a plotagem em diagrama de dispersão segundo proposto por Bland e Altman ${ }^{(24)}$ para a verificação da concordância entre as medidas obtidas pelos dois métodos: questionário de atividade física e frequencímetro.

\section{Aspectos éticos}

As gestantes foram informadas sobre os procedimentos e objetivos do estudo e quando concordavam em participar, assinavam o termo de consentimento livre e esclarecido, aprovado nos Comitês de Ética da faculdade de saúde pública da universidade de São Paulo e dos serviços de saúde.

\section{RESULTADOS}

\section{Características da população estudada e comparação com as perdas}

Foram contatadas 115 mulheres no segundo trimestre de gestação seguindo os critérios de seleção, tendo ocorrido 32 recusas (28\%). Iniciaram o estudo 83 gestantes, no entanto 15 mulheres (18\%) desistiram. Os principais motivos apresentados para desistência foram o incômodo causado pelo uso do freqüencímetro e/ou falta de tempo para anotar o diário de atividades físicas. Quando as mulheres desistiam da participação do estudo em um intervalo da aplicação do primeiro questionário muito pequeno (inferior a 24h) o questionário não era reaplicado e estas mulheres foram consideradas perdas.

Houve variação no tamanho amostral nas análises estatísticas. A análise de reprodutibilidade do primeiro e segundo questionários abrangeu 68 mulheres, e entre o primeiro questionário e o questionário aplicado no pós-parto, 34 gestantes. Devido a falha e mal uso do equipamento (freqüencímetro) a avaliação da validade foi realizada com dados de 38 gestantes.

A Tabela 1 mostra o perfil das mulheres estudadas em comparação com as perdas de seguimento.

Nas mulheres estudadas a idade variou de 18 a 33 anos. A escolaridade média foi de nove anos. Do total de mulheres estudadas, 22,1\% moravam sem companheiro. Dentre aquelas com companheiro cerca de um terço estava nesta situação a menos de dois anos.

Mais da metade das gestantes trabalhava fora de casa e dois terços dessas estava satisfeita com o trabalho. Quando indagadas sobre a modificação da atividade física no segundo trimestre de gestação, a maioria das gestantes $(70,6 \%)$ informou ter diminuído sua atividade física. Apenas quatro gestantes (5,8\%) referiram aumento da atividade física e um quarto da amostra referiu não ter alterado a atividade física. Com relação ao trabalho doméstico, mais da metade das mulheres contava com auxílio nessas tarefas. 
Tabela 1. Características das gestantes estudadas e das perdas de seguimento. São Paulo, 2004.

\begin{tabular}{|c|c|c|c|}
\hline $\begin{array}{l}\text { Características das } \\
\text { gestantes }\end{array}$ & $\begin{array}{c}\text { Amostra estudada } \\
(n=68)\end{array}$ & $\begin{array}{l}\text { Perdas } \\
(n=15)\end{array}$ & \\
\hline Variáveis contínuas & $\begin{array}{l}\text { Média e desvio } \\
\text { padrão }\end{array}$ & $\begin{array}{c}\text { Média e desvio } \\
\text { padrão }\end{array}$ & $p^{a}$ \\
\hline Idade (anos) & $26,9 \pm 6,1$ & $24,1 \pm 5,4$ & 0,11 \\
\hline $\begin{array}{l}\text { Escolaridade (anos completos } \\
\text { de estudo) }\end{array}$ & $9,5 \pm 3,1$ & $8,3 \pm 2,7$ & 0,17 \\
\hline $\begin{array}{l}\text { Número de pessoas no } \\
\text { domicílio }\end{array}$ & $3,7 \pm 1,6$ & $4,0 \pm 2,3$ & 0,61 \\
\hline Peso pré-gestacional (kg) & $63,2 \pm 12,5$ & $62,0 \pm 17,1$ & 0,78 \\
\hline Altura $(\mathrm{cm})$ & $162,7 \pm 6,0$ & $162,1 \pm 6,8$ & 0,75 \\
\hline $\begin{array}{l}\text { Peso }(\mathrm{kg}) \text { no momento da } \\
\text { entrevista }\end{array}$ & $69,5 \pm 13,1$ & $66,8 \pm 16,3$ & 0,51 \\
\hline Renda total familiar & $1327,2 \pm 1924,3$ & $1104,6 \pm 831,0$ & 0,71 \\
\hline IMC pré-gestacional (kg/m²) & $23,6 \pm 6,5$ & $24,0 \pm 4,4$ & 0,75 \\
\hline Variáveis categóricas & $\%$ & $\%$ & $p^{b}$ \\
\hline Planejaram a gravidez & 32,4 & 26,7 & 0,53 \\
\hline Queriam ter o filho & 58,8 & 60,0 & 0,63 \\
\hline Primíparas & 44,1 & 33,3 & 0,63 \\
\hline Fumavam durante a gravidez & 9,2 & 18,2 & 0,34 \\
\hline $\begin{array}{l}\text { Trabalharam fora de casa } \\
\text { durante a gravidez }\end{array}$ & 52,9 & 46,2 & 0,88 \\
\hline
\end{tabular}

a: Teste t; b: Qui-quadrado ou Teste exato de Fisher

\section{Análise de reprodutibilidade do questionário}

As informações quanto ao tempo despendido nas diferentes dimensões de atividade física estimado pelo questionário, nos três momentos, são apresentadas na Tabela 2.

A aparente redução no tempo despendido após o parto nas três intensidades agrupadas e a elevação da caminhada, no período pósparto não se confirmou quando a comparação foi feita para as 34 gestantes que responderam os três instrumentos.

Os resultados da análise de reprodutibilidade para as variáveis categóricas com uma semana de intervalo (Questionário 1 × 2) e no pós-parto imediato (Questionário 1×3) são apresentadas na Tabela 3.

Tabela 2. Valores medianos e quartis do tempo despendido em diferentes dimensões da atividade física. São Paulo, 2004

\begin{tabular}{|c|c|c|c|}
\hline VARIÁVEIS & $\begin{array}{l}\text { Questionário } 1 \\
(n=68)\end{array}$ & $\begin{array}{c}\text { Questionário } 2 \\
(\mathrm{n}=68)\end{array}$ & $\begin{array}{c}\text { Questionário } 3 \\
(n=34)\end{array}$ \\
\hline $\begin{array}{l}\text { Atividades sedentárias } \\
\text { (min/dia) }\end{array}$ & $\begin{array}{c}743,8 \\
(606,4 ; 929,4)\end{array}$ & $\begin{array}{c}756,4 \\
(621,6 ; 941,1)\end{array}$ & $\begin{array}{c}707,1 \\
(645,0 ; 926,3)\end{array}$ \\
\hline Atividades leves (min/dia) & $\begin{array}{c}284,21 \\
(141,1 ; 417,3)\end{array}$ & $\begin{array}{c}218,6 \\
(130,2 ; 378,3)\end{array}$ & $\begin{array}{c}185,7 \\
(116,4 ; 359,5)\end{array}$ \\
\hline $\begin{array}{l}\text { Atividades moderadas } \\
\text { (min/dia) }\end{array}$ & $\begin{array}{c}231,9 \\
(107,0 ; 480,7)\end{array}$ & $\begin{array}{c}230,4 \\
(106,1 ; 476,0)\end{array}$ & $\begin{array}{c}132,0 \\
(61,2 ; 432,7)\end{array}$ \\
\hline $\begin{array}{l}\text { Caminhada } \\
\text { (min/dia) }\end{array}$ & $\begin{array}{c}57,1 \\
(25,6 ; 117,1)\end{array}$ & $\begin{array}{c}51,7 \\
(28,7 ; 145,8)\end{array}$ & $\begin{array}{c}79,8 \\
(44,0 ; 121,8)\end{array}$ \\
\hline $\begin{array}{l}\text { Permanência em pé parada } \\
\text { (min/dia) }\end{array}$ & $\begin{array}{c}114,5 \\
(65,9 ; 180,0)\end{array}$ & $\begin{array}{c}95,5 \\
(58,04 ; 158,9)\end{array}$ & $\begin{array}{c}75,3 \\
(34,5 ; 143,8)\end{array}$ \\
\hline Postura agachada (min/dia) & $\begin{array}{c}7,1 \\
(0,5 ; 17,1)\end{array}$ & $\begin{array}{c}5,4 \\
(1,0 ; 17,14)\end{array}$ & $\begin{array}{c}4,3 \\
(0,4 ; 8,6)\end{array}$ \\
\hline
\end{tabular}

A percepção subjetiva do nível de atividade física abrangeu cinco respostas: muito mais ativa, mais ativa, tão ativa quanto, menos ativa, muito menos ativa comparando com outras gestantes. A concordância foi boa quando a comparação foi feita durante a gestação e menor antes de engravidar. Resultados satisfatórios foram também verificados para ajuda nas tarefas domésticas, realização de atividade física que aumente a transpiração ou carregando objetos.

Tabela 3. Análise da reprodutibilidade do questionário de atividade física - variáveis categóricas. São Paulo, 2004.

\begin{tabular}{|c|c|c|c|c|c|}
\hline \multirow{2}{*}{\multicolumn{2}{|c|}{ Questões: }} & \multicolumn{2}{|c|}{ Questionário $1 \times 2(n=68)$} & \multicolumn{2}{|c|}{ Questionário $1 \times 3(n=34)$} \\
\hline & & Kappa* & $\mathrm{IC}_{95 \%}$ & Kappa* & $\mathrm{IC}_{95 \%}$ \\
\hline \multirow{5}{*}{$\begin{array}{c}\text { Você } \\
\text { pratica } \\
\text { alguma } \\
\text { atividade } \\
\text { física: }\end{array}$} & Na qual transpire & 0,56 & $0,32-0,79$ & 0,46 & $0,18-0,74$ \\
\hline & $\begin{array}{l}\text { Carregando obje- } \\
\text { tos pesados }\end{array}$ & 0,63 & $0,40-0,87$ & 0,66 & $0,32-0,99$ \\
\hline & Esportiva & 0,41 & $0,17-0,64$ & - & \\
\hline & Vigorosa & 0,32 & $0,09-0,55$ & 0,53 & $0,19-0,86$ \\
\hline & Moderada & 0,29 & $0,07-0,52$ & 0,08 & $-0,02-0,39$ \\
\hline \multirow{2}{*}{$\begin{array}{c}\text { Quão } \\
\text { ativa } \\
\text { você se } \\
\text { considera: }\end{array}$} & $\begin{array}{l}\text { Comparando com } \\
\text { outras gestantes }\end{array}$ & $0,64^{\S}$ & $0,40-0,88$ & $0,64^{5}$ & $0,30-0,98$ \\
\hline & $\begin{array}{l}\text { Antes de engra- } \\
\text { vidar }\end{array}$ & $0,43^{\S}$ & $0,19-0,66$ & $0,42^{5}$ & $0,09-0,76$ \\
\hline \multicolumn{2}{|c|}{$\begin{array}{l}\text { Alguém ajuda nas tarefas } \\
\text { domésticas }\end{array}$} & 0,76 & $0,52-0,99$ & 0,70 & $0,36-1,00$ \\
\hline
\end{tabular}

${ }^{5}$ Kappa ponderado

As questões referentes à prática de atividade física esportiva, vigorosa e moderada, adaptadas do IPAQ versão longa, apresentaram valores abaixo do esperado, demonstrando dificuldade de compreensão pelas gestantes estudadas.

Quanto à avaliação das variáveis de atividade física segundo intensidades de esforço diário, a análise de reprodutibilidade também demonstrou bons resultados com uma semana de intervalo $(n=68)$ e no pós-parto imediato ( $n=34)$, conforme pode ser observado na Tabela 4. Entretanto, a postura, identificada como a permanência em pé e agachada obteve valores baixos de correlação intraclasse.

Tabela 4. Análise da reprodutibilidade do questionário de atividade física, mediante o coeficiente de correlação intraclasse (CCI), entre os três questionários. São Paulo, 2004.

\begin{tabular}{|c|c|c|c|c|}
\hline \multirow{2}{*}{ VARIÁVEIS } & \multicolumn{2}{|c|}{ Questionários 1 × 2} & \multicolumn{2}{|c|}{ Questionários $1 \times 3$} \\
\hline & $\mathrm{CCl}$ & $\mathrm{IC}(95 \%)$ & $\mathrm{CCl}$ & $\mathrm{IC}(95 \%)$ \\
\hline $\begin{array}{l}\text { Tempo gasto em atividades sedentá- } \\
\text { rias (min/dia) }\end{array}$ & 0,81 & $0,70-0,88$ & 0,84 & $0,71-0,91$ \\
\hline $\begin{array}{l}\text { Tempo gasto em atividades leves } \\
\text { (min/dia) }\end{array}$ & 0,85 & $0,76-0,91$ & 0,83 & $0,70-0,91$ \\
\hline $\begin{array}{l}\text { Tempo gasto em atividades modera- } \\
\text { das (min/dia) }\end{array}$ & 0,75 & $0,60-0,85$ & 0,73 & $0,52-0,84$ \\
\hline $\begin{array}{l}\text { Tempo em atividades em postura } \\
\text { agachada (min/dia) }\end{array}$ & 0,54 & $0,20-0,74$ & 0,71 & $0,65-0,90$ \\
\hline Tempo gasto em caminhada (min/dia) & 0,80 & $0,67-0,88$ & 0,80 & $0,65-0,90$ \\
\hline $\begin{array}{l}\text { Tempo em atividades em postura em } \\
\text { pé parada (min/dia) }\end{array}$ & 0,56 & $0,29-0,73$ & 0,74 & $0,54-0,86$ \\
\hline
\end{tabular}


Os resultados da reprodutibilidade do questionário foram melhores na avaliação das variáveis quantitativas quando comparados aos obtidos para as variáveis qualitativas nas quais há maior dependência da percepção subjetiva.

\section{Análise de validade do questionário}

As informações quanto ao tempo despendido nas diferentes dimensões de atividade física, estimado pelos dois instrumentos questionário e freqüencímetro são apresentadas na Tabela 5.

Tabela 5. Valores medianos e quartis do tempo despendido em diferentes dimensões da atividade física (questionário e frequêncimetro). São Paulo, 2004.

\begin{tabular}{l|c|c}
\hline \multicolumn{1}{c|}{ VARIÁVEIS } & $\begin{array}{c}\text { Questionário 1 } \\
(\mathbf{n = 6 8 )}\end{array}$ & $\begin{array}{c}\text { Freqüencímetro } \\
\mathbf{( n = 3 8 )}\end{array}$ \\
\hline Atividades leves (min/dia) & $284,21(141,07 ; 417,32)$ & $333,30(268,55 ; 454,74)$ \\
\hline Atividades moderadas (min/dia) & $231,93(106,98 ; 480,71)$ & $214,75(148,50 ; 340,21)$ \\
\hline
\end{tabular}

As estimativas de atividades sedentárias não foram adequadas quando se comparou o questionário e o freqüencímetro como medida padrão, visto que o equipamento apresenta falhas de registro quando a mulher está em decúbito lateral, conforme relatado pelas gestantes e constatado no trabalho de campo.

Não houve diferença significativa entre os valores obtidos pelo questionário e freqüencímetro tanto para atividades leves quanto para moderadas.

Os diagramas de dispersão com a plotagem dos valores médios (abscissa) de tempo despendido em atividades leves (Figura 1) e moderadas (Figura 2), pelas diferenças individuais dos instrumentos (ordenadas) são ilustrados a seguir. Esse procedimento de análise proposto por Bland e Altman ${ }^{(24)}$ permite visualizar as diferenças médias e os limites extremos de concordância ( \pm 2 DP da diferença) apresentados na comparação dos instrumentos.

A média das diferenças obtidas entre os valores obtidos pelo questionário e pelo freqüencímetro se aproximaram de zero nas atividades leves. Resultado similar foi obtido para a média das diferenças considerando as atividades moderadas com valor próximo de 44 minutos por dia.

Os limites de concordância foram amplos tanto para as atividades leves quanto moderadas, aproximando-se de sete e 11 horas por dia

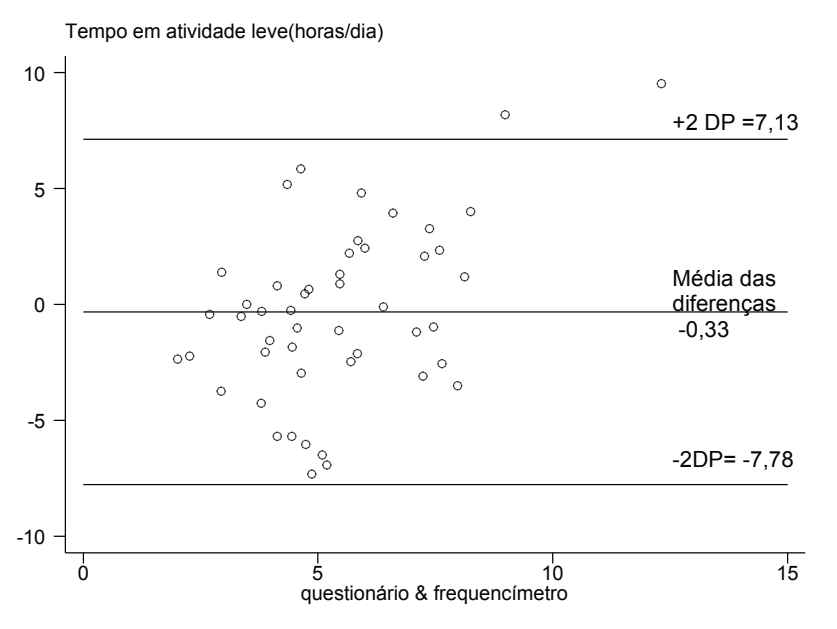

Figura 1. Análise comparativa do tempo em atividades leves entre o questionário e freqüencímetro.

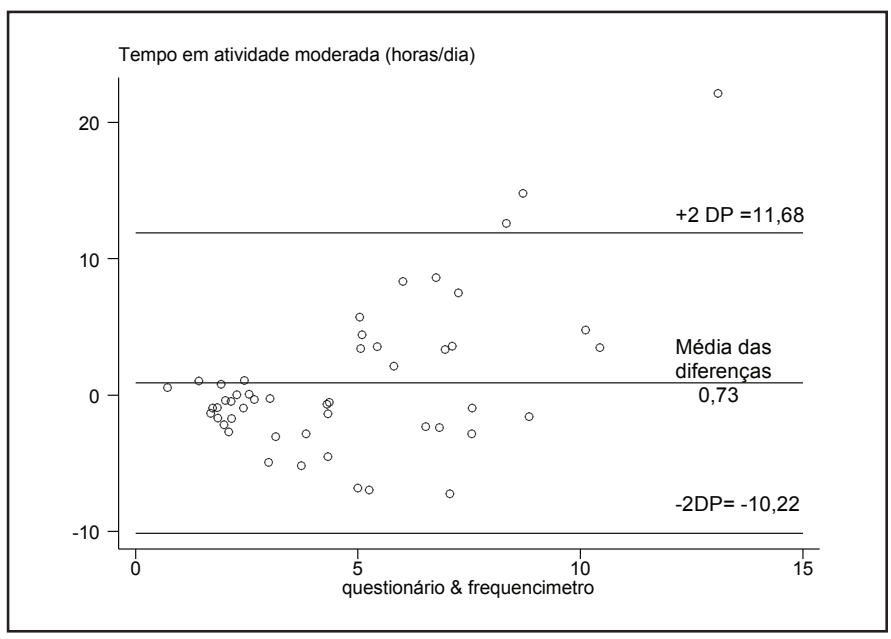

Figura 2. Análise comparativa do tempo em atividades moderadas entre o questionário e freqüencímetro.

respectivamente. Além desse fator a maior dispersão dos dados das atividades moderadas, com o aumento da magnitude das medidas indica menor precisão para valores mais elevados. Nas atividades leves, observa-se dispersão adequada dos dados.

\section{DISCUSSÃO}

Os questionários destacam-se como o instrumento mais acessível para avaliação da atividade física habitual, pela facilidade de aplicação em grandes grupos, pelo menor custo e pela possibilidade de coletar informações com relação ao tipo e ao contexto em que são realizadas, sendo instrumentos importantes em estudos epidemiológicos. No entanto, as características das informações que buscam coletar/obter podem sofrer maior variação, pois dependem do relato dos indivíduos e, portanto, da memória e da percepção subjetiva em relação ao esforço e duração da atividade entre outros aspectos. Assim, quando comparado com outros métodos, podem demonstrar maiores problemas quando avaliado quanto à reprodutibilidade e validade de seus resultados.

A utilização de questionários auto-preenchidos tem acurácia reduzida em mulheres de menor nível educacional(17). Para minimizar erros de medida foi elaborado manual de instruções detalhado e realizado treinamento das entrevistadoras visando a correta aplicação do questionário. Ainda assim, possível viés de informação pode ocorrer na aplicação do questionário.

Estudos de validação demandam tempo substancial e cooperação por parte dos participantes, tendo em geral elevado número de recusas. Nesse estudo, o percentual de recusas foi igual a 28\%. Deve-se considerar a possibilidade de as mulheres que concordaram em participar diferenciem-se da população em geral com relação à acurácia dos níveis de atividade física reportados. A perda de seguimento foi igual a 18\% não havendo diferenças significativas entre o grupo estudado e as perdas.

Outro fator importante na análise dos resultados é a modificação da atividade física habitual que ocorre nesse período, com três quartos das mulheres referindo alguma modificação, quando questionadas no segundo trimestre de gestação. Esse fato dificulta a mensuração das atividades físicas cotidianas, uma vez que, segundo Misra et al. ${ }^{(8)}$ modificações importantes ocorrem nos dois primeiros trimestres de gestação.

Os erros em replicar as estimativas do questionário podem ter diversas fontes. Primeiramente o curto intervalo de tempo entre as 
aplicações do questionário (cerca de uma semana) pode ter influenciado a memória da mulher e resultar em melhores coeficientes de correlação. Por outro lado, a utilização de equipamento e preenchimento do diário de atividades físicas podem ser fatores modificadores, tanto da percepção da gestante quanto no tocante à modificação das próprias atividades diárias. Para minimizar esses problemas foi também analisado o período pós-parto.

É importante mencionar a enorme dificuldade na análise dos diários de atividades físicas, ainda que tenham sido excluídas mulheres não alfabetizadas, devido ao preenchimento incorreto. A listagem de diferentes atividades no mesmo período de tempo foi freqüente. Este fato dificultou a quantificação exata da duração, início e término de cada atividade e impossibilitou a utilização do diário como parâmetro na interpretação da freqüência cardíaca para cada atividade realizada.

O Compêndio de Atividades Físicas(22), proposto como sistema de classificação e padronização das intensidades segundo o equivalente metabólico de repouso (MET), não foi desenvolvido para determinar com precisão o custo energético individual das atividades físicas. No entanto, verifica-se que muitos profissionais e pesquisadores o utilizam para expressar o gasto energético em relação ao peso corporal e realizar prescrição de exercício. Dentre as críticas quanto à sua utilização para estimativa do gasto energético, encontra-se a superestimação em grupo heterogêneo de indivíduos ${ }^{(25)}$ ou a subestimação em indivíduos obesos ${ }^{(26)}$. Por esse motivo, a presente análise utilizou o Compêndio como uma estratégia de classificação das atividades físicas, apenas para analisar o tempo despendido nas várias dimensões de atividade física.

Os resultados de reprodutibilidade encontrados com relação às questões qualitativas do questionário em três momentos foram satisfatórios somente para três questões: carregar objetos pesados, como percebia seu nível de atividade física durante a gestação e ajuda nos serviços domésticos. Os coeficientes de concordância Kappa entre Q1 e Q2 situaram-se na faixa de classificação médio para bom, de 0,40 a 0,75 (Cohen, 1960)(23). Estes dados estão de acordo com os resultados apresentados por Wildshut et al.(15), exceção feita às questões subjetivas com desempenho insatisfatório em seu estudo.

No tocante às variáveis quantitativas, a análise de correlação intraclasse apresentou bons resultados na reprodutibilidade do questionário. Entretanto atividades agrupadas como permanência em pé apresentaram valores abaixo do esperado. Ainda que a maneira de agrupar as diversas atividades diárias tenha sido diferente, os resultados obtidos por Schmidt et al.(17) nas atividades agrupadas foram semelhantes, variando de 0,76 para atividades habituais a 0,86 para ocupacionais.

Muitos estudos utilizam inapropriadamente o coeficiente de correlação produto momento como indicador de concordância entre dois instrumentos. Assim o fizeram, os recentes estudos de validação de questionários de atividade física em gestantes, de Chasan-Taber et al. ${ }^{(16,17)}$, os quais apresentam bons coeficientes de correlações de Pearson e Spearman. No entanto, a relação linear não considera e diferencia a inclinação dos pontos, nem diferenças sistemáticas entre os instrumentos que podem afetar substancialmente a concordân$\mathrm{cia}^{(27)}$. A análise proposta por Bland e Altman ${ }^{(24)}$ é mais adequada, pois considera os valores médios obtidos em cada método em relação à diferença obtida entres eles, verificando o grau de concordância entre os dados obtidos.

Pequena diferença foi verificada na comparação entre as médias dos valores do questionário com a freqüência cardíaca, cujos valores se aproximavam de zero, tanto para as atividades leves quanto moderadas. Porém, a verificação dos limites de concordância $( \pm 2$ desvios padrão) indica grande variação, mais de 10 horas diárias para atividades moderadas e sete horas para as atividades leves, representando quase um terço do total diário. Ainda que a maioria dos pontos encontre-se dentro dos limites, esses valores apontam para variabilidade individual demasiadamente elevada nas medidas do tempo despendido nas duas dimensões da atividade física entre os instrumentos.

Em relação à disposição gráfica, dispersão adequada dos dados é observada nas atividades leves indicando que as diferenças não variaram de maneira sistemática em toda a faixa de medida. Por outro lado, os dados obtidos nas atividades moderadas sugerem maior variabilidade. Além disso, a inclinação dos pontos indica tendência a aumento da diferença à medida que aumenta o tempo despendido.

Esse resultado discordante entre métodos também foi encontrado por Stein et al.(28) ao analisarem 56 mulheres, com o objetivo de descrever padrões de gasto energético durante a gestação e verificar a validade concorrente de três métodos de estimativa. Os autores verificaram que a estimativa do gasto energético subestimou os valores obtidos com o sensor de movimentos (acelerômetro) e superestimou os valores baseados no recordatório de atividades físicas quando comparados com a estimativa da freqüência cardíaca mensurada, em ambos grupos e períodos da gestação. A análise estatística apresentou coeficientes de correlação de Pearson (pairwise) entre 0,07 e 0,81. Segundo os autores, os três métodos são sensíveis às variações do gasto energético durante cada atividade. O recordatório de atividade física e o sensor de movimento (acelerômetro), bastante utilizados em estudos epidemiológicos para categorizar a atividade física de gestantes, apresentaram diferenças em relação à freqüência cardíaca.

A opção por um método de referência é o ponto crítico na análise das informações para a validação de um instrumento, considerando que esse deverá mensurar com a maior acurácia possível as várias dimensões da atividade física. Os resultados confirmam a falta de um padrão ouro para mensuração da atividade física. Ainda que a mensuração da freqüência cardíaca tenha sido utilizada como uma medida objetiva, ela deve ser considerada medida indireta da atividade física. A comparação das estimativas tanto no freqüêncímetro quanto no questionário são influenciadas por erros de medida. Adicionalmente, alteração da freqüência cardíaca com fatores externos ao esforço físico, tal como situações nas quais o indivíduo é tomado de sobressalto. Bem como, a solicitação de retirada do equipamento em atividades na água e ao dormir somada às falhas de registro do equipamento em decúbito lateral também podem ter afetado os resultados. Outra falha detectada diz respeito à superestimação da medida com a proximidade de motor em funcionamento (carro/ônibus).

Além disso, a freqüência cardíaca em gestantes apresenta significativas alterações fisiológicas inerentes à gestação como o aumento do volume plasmático. Os cuidados tomados no presente estudo incluíram a mensuração no mesmo trimestre da gestação, utilização de equação proposta para gestantes no mesmo período ${ }^{(29)}$ e seleção de equipamento (Polar) acurado ${ }^{(30)}$.

Ao analisar o questionário proposto por Chasan-Taber et a ${ }^{(16)}, \mathrm{o}$ auto-preenchimento restringe a utilização em mulheres de menor escolaridade, parcela expressiva da nossa população. Diferenças sócioeconômicas podem impossibilitar a sua utilização, visto que as categorias estabelecidas a priori no questionário subestimam a realização, por exemplo, de atividades domésticas pesadas e leves. A categoria mais elevada do questionário corresponde a três ou mais horas por 
semana, e as gestantes de nosso estudo apresentaram mediana igual a quatro e seis horas por semana.

Schmidt et al. ${ }^{(17)}$ utilizaram o Kaiser Physical Activity Questionnaire, questionário previamente validado para mulheres para avaliar a atividade física total por intermédio de um índice. Essa metodologia reduz a possibilidade de proposições e recomendações específicas para atividade física. Em estudo anterior no qual atividades em pé puderam ser detalhadas, identificou-se associação específica da atividade de lavar roupa com o peso inadequado ao nascer ${ }^{(31)}$.

As análises do presente estudo parecem assegurar a reprodutibilidade do questionário de atividade física para gestantes usuárias de serviços públicos de saúde com características semelhantes a amostra estudada. Entretanto, com relação à validação, a comparação com os resultados obtidos pelo freqüencímetro - instrumento disponível na época da coleta de dados - não mostrou graus de concordância adequados. São necessários estudos que utilizem metodologia estatística semelhante à aqui empregada e instrumentos mais adequados de medida de atividade física. É possível supor que o questionário de atividades físicas proposto por esse estudo possa ter resultados satisfatórios quando comparado a outros métodos de medida mais precisos, tais como acelerômetro triaxial ou água duplamente marcada. Mais estudos são necessários para a identificação de metodologias adequadas de mensuração da atividade física durante a gestação em estudos epidemiológicos.

Todos os autores declararam não haver qualquer potencial conflito de interesses referente a este artigo.

\section{REFERÊNCIAS BIBLIOGRÁFICAS}

1. WHO. 2002 world report: reducing risks, promoting healthy life. Geneva: WHO; 2002.

2. Pate RR, Pratt M, Blair SN, Haskell WL, Macera CA, Bouchard C, et al. Physical activity and public health. A recommendation from the Centers for Disease Control and Prevention and the American College of Sports Medicine. Jama 1995; 273: 402-7.

3. Matsudo SM, Matsudo VR, Araujo TL, Andrade DR, Andrade EL, de Oliveira LC, et al. The Agita Sao Paulo Program as a model for using physical activity to promote health. Rev Panam Salud Publica 2003; $14: 265-72$.

4. BRASIL. Inquérito domiciliar sobre comportamentos de risco e morbidade referida de doenças e agravos não transmissíveis: Brasil, 15 capitais e Distrito Federal, 2002-2003. Rio de Janeiro: Ministério da Saúde. Secretaria de Vigilância em Saúde. Secretaria de Atenção à Saúde. Instituto Nacional de Câncer. Coordenação de Prevenção e Vigilância. ; 2004.

5. Artal R, O'Toole M. Guidelines of the American College of Obstetricians and Gynecologists for exercise during pregnancy and the postpartum period. Br J Sports Med 2003; 37:6-12; discussion 12.

6. Hatch MC, Shu XO, McLean DE, Levin B, Begg M, Reuss L, et al. Maternal exercise during pregnancy, physical fitness, and fetal growth. Am J Epidemiol 1993; 137: 1105-14.

7. Pivarnik JM. Potential effects of maternal physical activity on birth weight: brief review. Med Sci Sports Exerc 1998; 30: 400-6.

8. Misra DP, Strobino DM, Stashinko EE, Nagey DA, Nanda J. Effects of physical activity on preterm birth. Am J Epidemiol 1998; 147: 628-35.

9. Kramer MS. Regular aerobic exercise during pregnancy. Cochrane Database Syst Rev 2000: CD000180.

10. Washburn RA, Ficker JL. Physical Activity Scale for the Elderly (PASE): the relationship with activity measured by a portable accelerometer. J Sports Med Phys Fitness 1999; 39: 336-40.

11. Washburn RA, Jacobsen DJ, Sonko BJ, Hill JO, Donnelly JE. The validity of the Stanford Seven-Day Physical Activity Recall in young adults. Med Sci Sports Exerc 2003; 35: 1374-80.

12. Boreham C, Riddoch C. The physical activity, fitness and health of children. J Sports Sci 2001; 19: 915-29.

13. Pardini R MS, Araújo T, Matsudo V, Andrade E, Braggion G, Andrade D, Oliveira L, Figueira JR A, Raso V. Validação de questionário internacional de nível de atividade física (IPAQ - versão 6): um estudo piloto em adultos jovens brasileiros. Rev Bras Cien Mov 2002; 10: 41-50

14. Florindo A, Latorre MRDO. Validation and reliability of the Baecke questionnaire for the evaluation of habitual physical activity in adult men. Rev Bras Med Esporte 2003; 9: 129-35.

15. Wildschut HI, Harker LM, Riddoch CJ. The potential value of a short self-completion questionnaire for the assessment of habitual physical activity in pregnancy. J Psychosom Obstet Gynaecol 1993: 14: 17-29.
16. Chasan-Taber L, Schmidt MD, Roberts DE, Hosmer D, Markenson G, Freedson PS. Development and validation of a Pregnancy Physical Activity Questionnaire. Med Sci Sports Exerc 2004; 36: 1750-60.

17. Schmidt MD, Freedson PS, Pekow P, Roberts D, Sternfeld B, Chasan-Taber L. Validation of the Kaiser Physical Activity Survey in pregnant women. Med Sci Sports Exerc 2006; 38: 42-50.

18. Hulley S, Cummings S. Designing Clinical Research: an epidemiological approach. Baltimore: Willians \& Wilkins; 1998.

19. Taylor HL, Jacobs DR, Jr., Schucker B, Knudsen J, Leon AS, Debacker G. A questionnaire for the assessment of leisure time physical activities. J Chronic Dis 1978; 31: 741-55.

20. Craig CL, Marshall AL, Sjostrom M, Bauman AE, Booth ML, Ainsworth BE, et al. International physical activity questionnaire: 12-country reliability and validity. Med Sci Sports Exerc 2003; 35: 1381-95.

21. Tremblay MS, Shephard RJ, McKenzie TL, Gledhill N. Physical activity assessment options within the context of the Canadian Physical Activity, Fitness, and Lifestyle Appraisal. Can J Appl Physiol 2001; 26: 388-407.

22. Ainsworth BE, Haskell WL, Whitt MC, Irwin ML, Swartz AM, Strath SJ, et al. Compendium of physical activities: an update of activity codes and MET intensities. Med Sci Sports Exerc 2000; 32: S498-504.

23. Fleiss JL. Statistical methods for rates and proportions. 2 ed. New York: John Wiley \& Sons; 1981.

24. Bland JM, Altman DG. Statistical methods for assessing agreement between two methods of clinical measurement. Lancet 1986; 1: 307-10.

25. Byrne NM, Hills AP, Hunter GR, Weinsier RL, Schutz Y. Metabolic equivalent: one size does not fit all. J Appl Physiol 2005; 99: 1112-9.

26. Leenders N, Sherman WM, Nagaraja HN. Comparisons of four methods of estimating physical activity in adult women. Med Sci Sports Exerc 2000; 32: 1320-6.

27. Hallal PC, Victora CG. Reliability and validity of the International Physical Activity Questionnaire (IPAQ). Med Sci Sports Exerc 2004; 36: 556.

28. Stein AD, Rivera JM, Pivarnik JM. Measuring energy expenditure in habitually active and sedentary pregnant women. Med Sci Sports Exerc 2003; 35: 1441-6.

29. Pivarnik JM, Stein AD, Rivera JM. Effect of pregnancy on heart rate/oxygen consumption calibration curves. Med Sci Sports Exerc 2002; 34: 750-5.

30. Achten J, Jeukendrup AE. Heart rate monitoring: applications and limitations. Sports Med 2003; 33: 517-38

31. Takito MY, Benicio MH, Latorre Mdo R. [Maternal posture and its influence on birthweight]. Rev Saude Publica 2005: 39: 325-32. 